\title{
Controle de Admissão para Redes em Malha de Última Milha
}

\author{
Edgar Piacentini, Anelise Munaretto e Mauro Fonseca
}

\begin{abstract}
Resumo - As redes em malha IEEE 802.11 surgem como uma alternativa, em relação às redes cabeadas, para prover infraestrutura de acesso de última milha, fornecendo uma boa margem de confiabilidade, baixo custo, facilidade de instalação e expansão. Entretanto, devido a sua natureza compartilhada possuem uma capacidade limitada de recursos, sendo necessário um mecanismo efetivo de controle de tráfego que permita a utilização das mesmas em aplicações de tempo real. Esse estudo avalia a utilização de uma rede em malha como meio de acesso para localidades remotas conectadas via enlace de satélite, propondo um mecanismo centralizado de controle de admissão capaz de prover requisitos mínimos de QoS para o transporte de VoIP.
\end{abstract}

Palavras-chaves - Redes em Malha, Controle de Admissão, QoS, Satélite.

\begin{abstract}
IEEE 802.11 Wireless Mesh Networks emerge as an alternative to provide infrastructure for last mile access networks. The meshed topology provides good reliability, low cost, market coverage and scalability. However due to the shared nature of wireless medium, which limits the available network bandwidth, it is necessary to implement a effective load control scheme that enable the use of wireless mesh networks for real-time applications. This work proposes a centralized admission control scheme to provide acceptable QoS for realtime applications. We demonstrate the usability of our admission control scheme to provide VoIP access for remotes areas connected via satellite.
\end{abstract}

Keywords - Wireless Mesh, Admission Control, QoS, Satellite.

\section{INTRODUÇÃO}

A pesquisa de soluções de comunicação de baixo custo para redes de acesso em localidades remotas e de baixa densidade populacional é um tópico importante na área de telecomunicações. Prover serviços de voz e dados para essas localidades é uma tarefa bastante desafiadora, uma vez que as tecnologias convencionais de transporte, como fibra óptica e enlaces de rádio, são inviáveis devido aos elevados custos e dificuldades de implantação e manutenção.

Soluções que utilizam pares de cobre convencionais para a rede de acesso e enlaces de satélite como meio de transporte têm sido propostas e utilizadas com sucesso $[1,2]$. A solução apresentada em [1], utiliza um enlace de satélite para conectar um gateway de acesso remoto, o qual disponibiliza

Edgar José Piacentini e Mauro Fonseca, Programa de Pós-Graduação em Informática Aplicada, PUC-PR, Curitiba, Brasil, E-mails: edgar.piacentini@ppgia.pucpr.br, mauro.fonseca@ppgia.pucpr.br. Anelise Munaretto, Programa de Pós-Graduação em Engenharia Elétrica Informática Industrial, UTFPR, Curitiba, Brasil, E-mail: anelise@cpgei.cefetpr.br. pares telefônicos convencionais para os usuários das comunidades atendidas pelo Programa Geral de Metas para Universalização (PGMU) [3]. A maior parte das comunidades atendidas pelo PGMU localiza-se em áreas remotas e de difícil acesso, em muitos casos carentes de infra-estrutura básica. Atualmente, mais de 1500 localidades são atendidas por esta tecnologia no Brasil. Apesar da solução [1] ser reconhecida por sua inovação tecnológica, a utilização de pares de cobre convencionais como parte da solução eleva os custos de implantação e manutenção devido à baixa densidade demográfica das regiões onde é implantada.

A utilização de um enlace de satélite associado a uma rede em malha 802.11, como meio de acesso de última milha, surge como uma alternativa de custo reduzido, para comunidades remotas desprovidas de acesso a rede. A topologia em malha fornece uma boa margem de confiabilidade, baixo custo, facilidade de instalação e manutenção, podendo ser facilmente estendidas pela simples adição de dispositivos.

Entretanto, o uso de enlaces de satélite associados às redes de acesso em malha 802.11, representam um complicador para o transporte de serviços em tempo real sensíveis a atraso, perdas de pacotes e variação do atraso (jitter), como voz sobre IP (VoIP). Nesse estudo, propomos um controle de admissão centralizado para redes em malha capaz de limitar o número de fluxos aceitos, mantendo níveis satisfatórios de qualidade de voz. A partir de medidas efetuadas em um enlace de satélite e de simulações utilizando o NS-2 [9], concluímos a viabilidade de utilização do enlace de satélite associado a uma rede de acesso em malha 802.11.

Na seção 2 apresentamos um exemplo de topologia de rede em malha utilizando enlace de satélite. Na seção 3 avaliamos as restrições impostas pelo enlace de satélite na rede de acesso, definindo uma metodologia de cálculo da qualidade de voz utilizando o modelo E [5]. Na seção 4, discutimos os problemas relativos às redes em malha 802.11 , que devido a sua natureza compartilhada, possuem uma capacidade limitada de recursos. Na seção 5 propomos um controle de admissão capaz de limitar o acesso de usuários à rede, conforme requisitos mínimos de qualidade de voz. Na seção 6 são apresentados os resultados obtidos nos testes, sendo a conclusão apresentada na seção 7 .

\section{TOPOLOGIA UTILIZANDO ENLACE DE SATÉLITE}

A padronização e regulamentação do DVB-RCS (Digital Video Broadcasting Return Channel via Satellite) pelo ETSI (ETSI EN 301.790) permitiu a redução dos custos dos terminais VSAT, tornando viável a utilização do enlace de 
satélite para o transporte de serviços IP para regiões onde, devido a sua localização geográfica e ou topográfica desfavorável, as tecnologias tradicionais, como fibra ótica ou rádio são economicamente inviáveis.

A Figura 1 mostra um exemplo de rede de acesso em malha 802.11 conectada via um enlace de satélite. A rede de acesso é composta por roteadores sem fio estáticos, equipados com um ponto de acesso atuando como concentrador de tráfego, fornecendo conectividade para os dispositivos móveis dos usuários dentro de sua área de cobertura. Um dos roteadores sem fio atua como gateway entre o backbone em malha e a rede de transporte via satélite. Todos os recursos de rede necessários, como servidores, autenticadores VoIP e os gateways de acesso a rede de telefonia fixa, são acessíveis através do enlace de satélite.

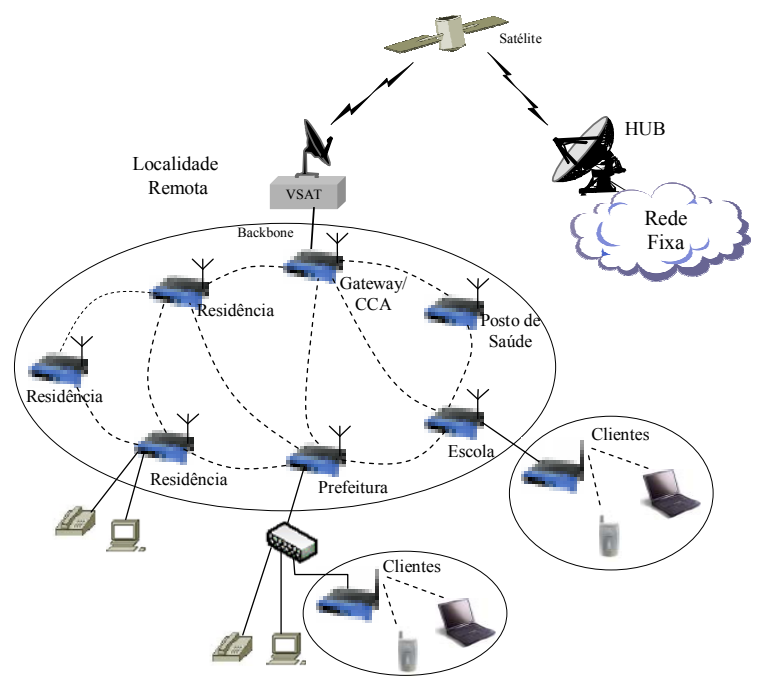

Figura 1 - Topologia utilizando enlace de satélite

No exemplo apresentado na Figura 1, cada cliente (residência, escola, posto de saúde, etc.), possui um roteador sem fio 802.11 que faz parte do backbone em malha. Os dispositivos desses clientes são conectados a um roteador do backbone através de uma interface Ethernet padrão. Os pontos de acesso são ajustados para utilizar um canal diferente do canal utilizado pelo backbone em malha, de forma a produzir a mínima interferência possível.

\section{RESTRIÇÕES IMPOSTAS PELO ENLACE DE SATÉLITE}

Os fatores que afetam a qualidade nas transmissões VoIP para as redes terrestres são geralmente conhecidos e bem entendidos. Entretanto, a utilização de enlaces de satélite geoestacionários para o transporte de serviços IP com requisitos de tempo real, requer algumas considerações adicionais. Um dos principais aspectos a ser considerado é o elevado atraso de propagação inerente dos sistemas via satélite. Outros aspectos importantes como custo de banda, jitter e perdas de pacotes podem ser mais bem administrados, mas também devem ser considerados.

$\mathrm{O}$ atraso e o jitter são os principais fatores que determinam a qualidade da telefonia sobre IP. O elevado atraso de propagação imposto pelo enlace de satélite restringe os limites disponíveis para a rede terrestre associada ao enlace. Entretanto, o atraso de propagação relativo a enlaces de satélite de um salto é aceitável e comercialmente utilizado há vários anos [1]. Um dos grandes inconvenientes do sistema DVB-RCS é a necessidade de dois saltos de satélite para conexão entre dois terminais VSAT [4]. Nesse caso o atraso de propagação assume valores muito elevados, comprometendo a qualidade de chamadas VoIP. Nesse estudo, consideramos a utilização de apenas um salto de satélite para conexão da rede de acesso em malha.

A tabela I mostra resultados de testes práticos efetuados na rede apresentada em [1]. Essas medidas são utilizadas como parâmetros para calcular as restrições que devem ser impostas na rede em malha de acesso. Os testes foram efetuados em condições normais de clima e temperatura, sendo que condições climáticas adversas podem provocar desvanecimento do sinal e eventuais variações nos resultados.

TABELA I. RESULTADO DOS TESTES NO SATÉLITE

\begin{tabular}{|c|r|r|}
\hline Medida & \multicolumn{1}{|c|}{ Média } & \multicolumn{1}{|c|}{ Desvio padrão } \\
\hline Atraso & $283 \mathrm{~ms}$ & $16 \mathrm{~ms}$ \\
\hline Jitter & $6 \mathrm{~ms}$ & $0,9 \mathrm{~ms}$ \\
\hline Perda de pacotes & $0,06 \%$ & $0,012 \%$ \\
\hline
\end{tabular}

Os resultados pressupõem que o terminal VSAT possui capacidade suficiente para alocar e garantir banda para as chamadas VoIP requisitadas.

Com o objetivo de avaliar o impacto do enlace de satélite associado à rede em malha de acesso, utilizamos a métrica proposta em [5], conhecida por Modelo E, para avaliar a qualidade das chamadas VoIP através do enlace de satélite (Figura 1). O Modelo E se baseia na idéia de que fatores psicológicos dentro de uma escala subjetiva são aditivos, sendo possível somá-los para se obter um grau da qualidade de voz em um sistema de comunicação [5]. Esse grau é dado pelo fator escalar R que varia de 0 a 100 , sendo que o valor 100 equivale à voz real. Conforme [5], é desejável que o valor de R seja superior a 70 .

$$
R=R_{0}-I_{s}-I_{d}-I_{e, e f f}+A
$$

Da equação (1), temos: $R_{0}$ representa a relação sinal-ruído básica, $I_{s}$ está associado ao fator de perdas simultâneas, $I_{d}$ ao fator de perdas associadas ao atraso fim-a-fim, $I_{e, \text { eff }}$ é o fator de perdas associadas ao equipamento utilizado e $A$ definido como o grau de tolerância que o usuário espera de uma determinada tecnologia. Conforme definido em [5], o valor de $A$ varia de 0 para telefonia fixa, até 20 para localidades de difícil acesso que necessitem de enlaces de satélite. Como $R_{0}$ e $I s$ são definidos em [5] e considerando $A$ igual a 20, a equação (1) pode ser expressa por:

$$
\begin{array}{ll}
R=102.36-0.023 * T_{a}-40 * \ln (1+10 * e) & \begin{array}{l}
\mathrm{p} / \mathrm{T}_{\mathrm{a}} \leq \\
175 \mathrm{~ms}
\end{array} \\
R=117.8-0.111 * T_{a}-40 * \ln (1+10 * e) & \mathrm{p} / \mathrm{T}_{\mathrm{a}}> \\
175 \mathrm{~ms}
\end{array}
$$

Da Equação (2) temos que: $T_{a}$ é dado pela soma dos atrasos relativos ao codec, ao buffer de jitter e a rede. 
A Figura 2 mostra a variação da qualidade da voz em relação ao atraso, dado pela equação (2). No cálculo, foi considerado um codec G.729 com o tamanho do buffer de jitter igual a $60 \mathrm{~ms}$ e taxa de perda de pacotes conforme Tabela I.

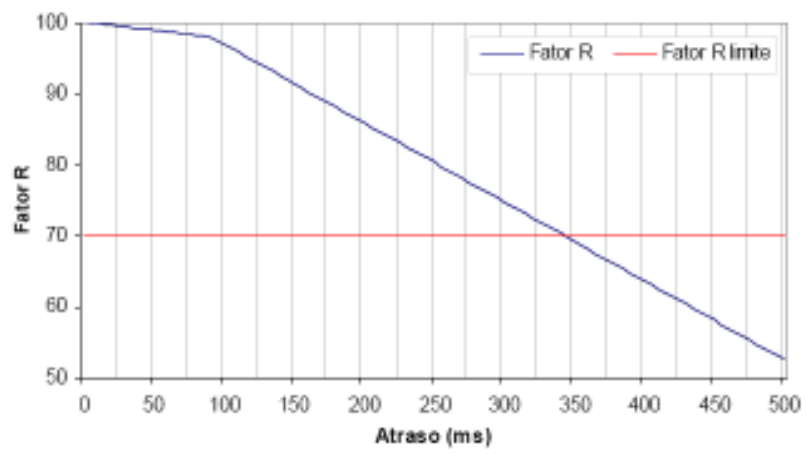

Figura 2 - Cálculo do Fator $\mathbf{R}$ relativo ao atraso

Baseado na análise do gráfico da Figura 2, podemos observar que para manter $R$ superior a 70, o atraso máximo não deve ser superior a aproximadamente $348 \mathrm{~ms}$. Se considerarmos o atraso do satélite dado na Tabela I, o atraso máximo que pode ser imposto pela rede de acesso fica em aproximadamente $65 \mathrm{~ms}$, não considerando possíveis perdas relativas à rede de acesso. No cálculo não foram considerados possíveis atrasos e perdas relativas à rede de telefonia fixa, por serem consideradas desprezíveis. Nas próximas sessões propomos um mecanismo de controle de admissão para redes em malha e verificamos, através de simulações, a viabilidade de utilização desse mecanismo em uma arquitetura via satélite.

\section{CONTROLE DE ADMISSÃO EM REDES EM MALHA 802.11}

O controle de admissão tem como função permitir que um novo fluxo entre na rede somente se os recursos disponíveis sejam maiores que os recursos requeridos pelo novo fluxo a ser admitido. Sem um mecanismo de controle de admissão, novos fluxos de dados podem entrar na rede mesmo que essa já esteja no seu limite de capacidade. Como conseqüência, tanto o novo fluxo quanto os fluxos já admitidos passam a sofrer problemas de atrasos e perdas, comprometendo os serviços que necessitam de requisitos de tempo real, como VoIP.

Para decidir se aceita o novo fluxo, o controle de admissão precisa estimar a quantidade de recursos disponíveis na rede e a quantidade de recursos que o novo fluxo vai ocupar. Em redes sem fio 802.11 um nó compartilha o meio com todos os outros nós que transmitem dentro de sua área de detecção de portadora (Carrier Sensing Range). A coordenação de acesso ao meio entre os nós dentro da área de detecção de portadora não é trivial. Eles podem ter uma visão diferente do meio, visto que os nós estão espacialmente distribuídos. Em outras palavras, um nó pode ser capaz de detectar a transmissão de outro nó, porém não conseguir decodificar os pacotes recebidos. Sendo assim dois nós que não podem se comunicar diretamente podem ainda disputar os mesmos recursos.
O mecanismo de controle de admissão deve assegurar que cada nó dentro da região de detecção de portadora, tenha recursos suficientes para acomodar o novo fluxo. Os recursos disponíveis em um nó dependem do link de transmissão, visto que cada link pode ter diferentes taxas de transmissão e de erro de pacotes. Para tornar o cálculo independente desses fatores, é usual medir a disponibilidade de recursos no canal em termos de tempo. A taxa de utilização do link, requerida por um fluxo, depende da taxa de geração dos pacotes, do tamanho dos pacotes, do fluxo e da taxa de transmissão do link. Entretanto, devido à auto-interferência dos fluxos, a utilização do link também é afetada pela fração de canal que o fluxo consome dos outros links vizinhos.

A garantia de QoS em redes 802.11 apresenta vários desafios e tem sido tema de várias pesquisas. Em [11] os autores propõem um controle de admissão para chamadas VoIP em uma rede em malha, baseado em um modelo de interferência. Os autores citam a interferência como o fator limitante do desempenho das redes em malha 802.11. Os estudos apresentados em $[10,13]$ procuram resolver os desafios relacionados ao controle de admissão em redes de múltiplos saltos. Os autores focam seus estudos no problema da comunicação com os nós vizinhos na área de detecção de portadora, propondo soluções que não podem ser implementadas com o hardware disponível atualmente. Em [10] os autores propõem um controle de admissão (Contention-aware Admission Control - CACP) para redes sem fio, que considera a natureza compartilhada do canal de rádio. Para fazer o controle de admissão, o mecanismo considera os recursos disponíveis não somente nos vizinhos mais próximos, mas também em todos os nós dentro da área de detecção de portadora. O mecanismo faz a estimativa dos recursos disponíveis em cada nó, pela monitoração passiva da quantidade de tempo que o canal é percebido como ocupado.

A maior parte dos mecanismos de controle de admissão propostos não consideram certas particularidades das redes em malha. Entre elas, podemos citar que ao contrário de uma rede ad-hoc, uma rede em malha apresenta topologia estável, exceto para eventuais falhas e adição de novos nós. Em uma rede em malha praticamente todo o tráfego é trocado entre um nó e um gateway, enquanto o tráfego nas redes ad-hoc flui entre pares de nós arbitrários. Neste artigo propomos um mecanismo de controle centralizado de admissão (CCA). Nosso estudo tem como base o mecanismo de controle de admissão proposto em [10] com as seguintes contribuições: Diferentemente do proposto em [10], onde o controle de admissão é efetuado em cada nó da rota, nosso mecanismo de controle de admissão é centralizado no gateway de acesso à rede de satélite, permitindo que o mesmo possa ser melhor gerenciado em termos de redundância à falhas. Isso resolve o problema da comunicação com os nós vizinhos, sendo que o mecanismo proposto permite também que os links operem em diferentes taxas de transmissão.

\section{MECANISMO DE CONTROLE DE AdMISSÃO}

O mecanismo de controle de admissão proposto parte do princípio que a topologia e a área de interferência de cada nó, são previamente conhecidas. O CCA mantém um perfil de cada nó da rede. O perfil inclui o tempo de ocupação do 
meio, percebido por cada nó e a taxa de transmissão de cada link. Esse perfil é gerado e mantido por mensagens de testes geradas pelos nós e enviadas ao CCA. O mecanismo proposto compreende a determinação da rota a ser seguida pelo fluxo, a estimativa dos recursos, o cálculo da interferência intrafluxo e a estimativa dos recursos disponíveis em cada nó da rede. $\mathrm{O}$ controle de admissão proposto decide se admite um novo fluxo baseado em duas considerações: primeira, se o nó e seus vizinhos têm recursos suficientes para acomodar o novo fluxo; e segunda, a admissão do novo tráfego não afeta negativamente os tráfegos já existentes. Essas duas considerações são avaliadas com a ajuda dos critérios operacionais descritos na seqüência.

\section{A. Determinação da Rota}

Quando um nó necessita admitir um novo fluxo, ele envia uma mensagem para o CCA contendo a origem, o destino, a taxa e o tamanho do pacote do fluxo a ser admitido. Com base nesses dados o CCA verifica se existe uma rota com recursos disponíveis e informa o nó solicitante.

Para calcular a rota, o CCA executa um algoritmo baseado no algoritmo de Djikstra (SPF), que verifica o caminho de menor custo entre a origem e o destino, com base na taxa de transmissão do link e no tempo de ocupação do canal. Em seguida o CCA verifica se o caminho calculado tem recursos suficientes para admitir o fluxo requisitado e retorna para o nó solicitante a rota a ser percorrida e um identificador do fluxo admitido.

Caso não haja recursos disponíveis na rota selecionada, o nó congestionado é desconsiderado e uma nova rota é calculada, sendo repetido o processo de verificação de recursos disponíveis para essa nova rota. No caso de não existir uma rota com recursos disponíveis, o CCA retorna uma mensagem informando a falta de recursos.

\section{B. Cálculo dos Recursos Disponíveis no Nó}

As medidas mais comuns utilizadas para avaliar a utilização de uma rede sem fio são baseadas no comprimento de filla, número de colisões, janela de utilização da camada MAC, atraso e tempo de ocupação do canal. As medidas de comprimento de fila, número de colisões e janela de utilização da camada MAC fornecem informações relevantes somente se a rede estiver congestionada [13], assim estes métodos não podem ser utilizados para estimar com nível suficiente de precisão a utilização da rede. O atraso é um dos métodos mais utilizados, entretanto, para redes sem fio onde a banda é limitada, a principal desvantagem é o overhead causado, uma vez que para se obter uma média confiável é necessário repetir várias vezes a medida.

O tempo de ocupação do canal é uma medida direta da utilização do canal. A avaliação do tempo de ocupação de um canal é tema de vários estudos [10,13]. Para estimar os recursos disponíveis em cada nó, o mecanismo proposto em [10] monitora passivamente a quantidade de tempo que o canal é percebido como ocupado. Esse tempo de ocupação inclui o tempo gasto em transmissão (Ttx), em recepção (Trx) e detecção de portadora (Tcs). Se o nó detecta uma portadora, ele percebe que o canal está ocupado, entretanto não consegue decodificar os pacotes. Pela medida da quantidade de tempo que o canal é percebido como transmitindo, recebendo ou detectando portadora, um nó pode determinar, não somente as transmissões que ocorrem dentro de sua área de recepção, mas também dentro de sua área de detecção de portadora. Pela monitoração da quantidade de tempo gasto em cada estado, é possível estimar a banda disponível no nó:

$$
B_{L}=(1-U) * \text { Capacidade Canal }
$$

Da Equação (4) temos: $U$ representa a somatória dos tempos de transmissão (Ttx), recepcão (Trx) e detecção de portadora (Tcs), em segundos [10].

No mecanismo proposto, utilizamos um método similar, onde os nós monitoram passivamente o canal, informando o CCA sobre o tempo de ocupação e a taxa de transmissão dos links. Essas informações são enviadas, através de uma mensagem, em intervalos de tempo regulares. Para reduzir o overhead, cada nó da rota, em direção ao CCA, adiciona a informação local ao pacote do nó anterior.

\section{Estimativa dos Recursos Consumidos pelo novo Fluxo}

Ao receber a requisição de um novo fluxo, o CCA faz uma estimativa do tempo que será consumido por esse fluxo em um intervalo de 1 segundo. Essa estimativa é feita com base no cálculo da capacidade teórica máxima do canal, através do tamanho e taxa de geração dos pacotes fornecidos pela mensagem de requisição e a taxa do link. No padrão IEEE 802.11 com o MAC utilizando a seqüência RTS-CTSDATA-ACK, o tempo gasto por um pacote de dados pode ser expresso como [8]:

$T_{p c}=T_{r t s}+T_{c t s}+\frac{L}{B}+T_{p l c p}+T_{a c k}+3 * T_{s i f s}+T_{d i f s}+T_{b o}$

Na expressão (4), os termos $L$ e $B$ são dependentes da taxa do link. $L$ é o tamanho do pacote incluindo o cabeçalho MAC e $B$ é a taxa do link. Os demais termos podem ser obtidos de [8]. Para o cálculo do tempo gasto para transmissão dos pacotes referentes a um fluxo em uma rede 802.11 b a expressão (4) pode ser expressa como:

$$
T_{\text {fluxo(s) }}=\left(1542+\frac{8 *(\operatorname{tam}+48)}{11}\right) * \frac{\operatorname{taxa}}{1000000}
$$

$\mathrm{Na}$ expressão (5), tam é o tamanho do pacote em bytes pertencente ao fluxo e a taxa representa o número de pacotes gerados pelo fluxo no intervalo de 1 segundo. Com base nessa estimativa e com o cálculo da interferência intra-fluxo, o CCA pode decidir se admite ou não o novo fluxo. O novo fluxo será admitido pela rota calculada se, para cada nó da rota e seus vizinhos dentro da região de detecção de portadora, a seguinte equação for satisfeita:

$$
T_{\text {fluxo }} * C C<\left(1-T_{\text {ocupado }}\right)
$$

$\mathrm{Na}$ expressão (6), $T_{\text {ocupado }}$ é informado pelo nó e $C C$ é calculado pelo CCA, como mostrado na seqüência. 


\section{Estimativa da Interferência Intra-Fluxo}

Devido à natureza compartilhada do meio, as transmissões sem fio consomem banda de todos os nós dentro da área de alcance de detecção de portadora do nó que está transmitindo. Além disso, múltiplos nós ao longo do caminho de um fluxo podem estar dentro do alcance de detecção de portadora uns dos outros, causando a chamada interferência intra-fluxo, na qual um nó da cadeia de encaminhamento terá os seus recursos divididos pelo número de nós situados na cadeia de encaminhamento que estejam situados dentro do seu alcance de detecção de portadora.

Para calcular a interferência intra-fluxo, é necessário conhecer a interferência de cada nó (Contention Count - CC), como definido em [10]. A banda efetiva consumida por um fluxo em cada nó é CC vezes a banda requisitada pela aplicação. Isso faz com que a interferência intra-fluxo gere um impacto significativo no consumo de banda do fluxo, sendo seu cálculo importante para um correto controle de admissão.

Como o CCA previamente conhece a topologia e a região de interferência de cada nó, ele consegue facilmente calcular o CC para cada nó afetado pelo fluxo na rota. Nosso mecanismo percorre cada nó da rota e calcula o $C C$ para o nó da rota e seus vizinhos dentro da região de detecção de portadora, conforme mostrado no pseudocódigo abaixo:

1. faça CC de todos os nós igual a zero

2. para $\mathrm{j} \leftarrow$ origem até (destino -1$)$, faça

3. para $\mathrm{i} \leftarrow 0$ até $\mathrm{n}$, faça

4. se nó(i) dentro da região de detecção de portadora nó(j), faça

5. $\mathrm{CC}($ nó(i) $)++$

6. fim-se

7. fim-para

8. fim-para

Note que o pseudocódigo assume que o próprio nó está dentro da área de detecção de portadora. Importante citar também que a região de interferência pode variar conforme a taxa de transmissão do link, entretanto essa taxa é informada juntamente com o tempo de ocupação ao CCA, permitindo que o mesmo assuma a região de interferência conforme a taxa do link.

\section{E. Decisão de Admissão}

O pseudocódigo abaixo mostra um resumo dos passos executados pelo CCA para admitir um novo fluxo.

1. nó solicita admissão de fluxo (origem, destino, taxa, tamanho)

2. algoritmo busca rota de menor custo entre origem e destino

3. se recursos disponíveis na rota, faça

4. reserva recursos e retorna a rota e o id do fluxo admitido

5. senão, faça

6. se outra rota disponível, faça

7. execute passo 3

8. senão, faça

9. retorna fluxo recusado

10. fim-se

11. fim-se

Sempre que um fluxo é terminado o nó informa, ao CCA, o identificador do fluxo terminado. Esse procedimento permite que o CCA, além de ter informações de tempo de ocupação e taxa do link de cada nó, também possua informações de todos os fluxos ativos na rede.

No pseudocódigo acima, o cálculo dos recursos disponíveis executado no passo três, é efetuado da seguinte forma: o CCA, baseado na taxa de geração de pacotes, no tamanho do pacote e na taxa do link, estima através da equação (4), o tempo gasto pelo fluxo em cada nó $T_{\text {fluxo. }} \mathrm{Na}$ seqüência o CCA, com base na taxa do link e informações previamente fornecidas, calcula o CC para cada nó da rota incluindo seus vizinhos na área de interferência de cada nó. Para o fluxo ser admitido na rota, todos os nós interferidos devem satisfazer a equação (6).

\section{RESULTADOS}

$\mathrm{O}$ algoritmo proposto foi implementado em linguagem $\mathrm{C}$ no ambiente do Dev-Cpp. Para simulação do tempo de ocupação de cada nó tomamos como base a equação (5), conforme descrito em [8]. Para validar os resultados do algoritmo, foram realizados testes comparativos com simulações realizadas no simulador NS-2 [9]. O NS-2 foi alterado para suportar roteamento estático, eliminando-se assim o overhead causado pelo descobrimento e manutenção das rotas. $\mathrm{O}$ alcance de transmissão foi configurado para $250 \mathrm{~m}$ e o alcance de detecção de portadora para $550 \mathrm{~m}$, com RTS/CTS habilitado. Os demais parâmetros foram configurados para o padrão IEEE 802.11 b. A simulação envolve nós separados por uma distância de $200 \mathrm{~m}$, permitindo que um nó se conecte apenas a seus vizinhos e interfira em no máximo dois nós. A Figura 3 mostra a topologia em cascata utilizada nas simulações. Os fluxos utilizados nas simulações foram configurados para CBR (Constant Bit Rate), com pacotes de 64 bytes e taxa de 50 pacotes por segundo, o que representa aproximadamente um fluxo VoIP com codec G.729 sem supressão de silêncio.

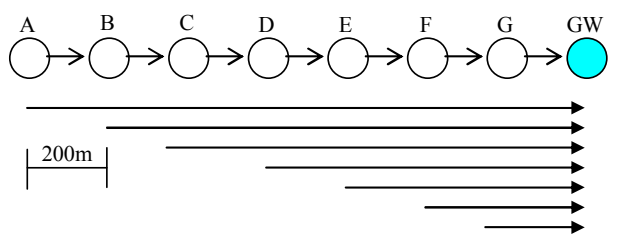

Figura 3 - Topologia utilizada na simulação

A Figura 4 mostra o atraso médio para doze e treze fluxos concorrentes, relativo a um salto ( $\mathrm{G}$ para $\mathrm{GW}$ na Figura 3 ). Assim que o fluxo 13 entra na rede, o atraso médio cresce drasticamente para valores acima de $70 \mathrm{~ms}$. Comportamento similar ocorre com a perda de pacotes e com o jitter. Esse comportamento se deve ao fato de que a capacidade nominal máxima do canal foi ultrapassada e a rede entrou em saturação. Somando-se os valores do atraso e da perda de pacotes, obtidos para 13 fluxos, aos valores obtidos na Tabela I, e tomando-se como base a equação (2), o valor do Fator R fica abaixo de 70, o que compromete a qualidade de voz para o caso em que a rede está saturada. 


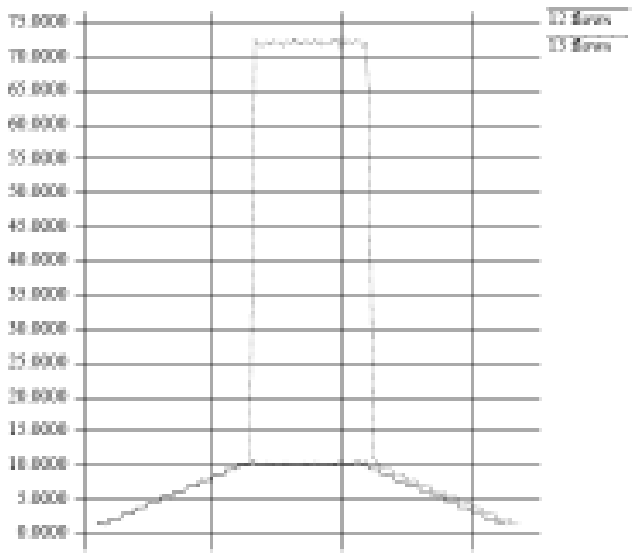

Figura 4 - Atraso para 12 e 13 fluxos com um salto

$\mathrm{Na}$ Tabela II são apresentados os resultados obtidos nas simulações para vários saltos, conforme a Figura 3. Para cada simulação, o número de fluxos é incrementado seqüencialmente até se obter a saturação, sendo computado nos resultados o número de fluxos suportados antes da saturação. Esse comportamento é também refletido pelo algoritmo proposto, como mostrado na última coluna da Tabela II.

TABELA II. RESULTADOS DA SIMULAÇÃO

\begin{tabular}{|c|c|c|c|c|c|c|}
\hline \multirow[b]{2}{*}{ Saltos } & \multicolumn{5}{|c|}{ NS-2 } & \multirow{2}{*}{$\begin{array}{c}\text { Algoritmo } \\
\text { Fluxos } \\
\text { admitidos }\end{array}$} \\
\hline & $\begin{array}{l}\text { Vazão máx } \\
\text { (kbps) }\end{array}$ & $\begin{array}{c}\text { Atraso } \\
(\mathrm{ms})\end{array}$ & $\begin{array}{c}\text { Perdas } \\
(\%)\end{array}$ & $\begin{array}{l}\text { Jitter } \\
(\mathrm{ms})\end{array}$ & $\begin{array}{l}\text { Nro. de } \\
\text { Fluxos }\end{array}$ & \\
\hline 1 & 317 & 10.3 & 0.2 & 2.9 & 12 & 12 \\
\hline 2 & 153 & 13.5 & 0.4 & 5.3 & 6 & 6 \\
\hline 3 & 107 & 12.9 & 0.5 & 6.2 & 4 & 4 \\
\hline 4 & 78 & 14.1 & 0.6 & 7.3 & 3 & 3 \\
\hline 5 & 70 & 12.4 & 0.5 & 5.6 & 2 & 2 \\
\hline 6 & 64 & 14.5 & 0.7 & 6.2 & 2 & 2 \\
\hline 7 & 62 & 16.3 & 0.8 & 6.5 & 2 & 2 \\
\hline
\end{tabular}

Foram realizadas simulações com o algoritmo implementado, utilizando-se fluxos (todos em direção ao gateway) gerados aleatoriamente, sendo que o número de fluxos admitidos simultaneamente variou de dois a doze, conforme esperado. Esse comportamento de doze chamadas simultâneas aparece quando todos os fluxos são admitidos em nós vizinhos de no máximo um salto do gateway.

A partir dos resultados apresentados na Tabela II e na Tabela I, calculamos o Fator $\mathrm{R}$ e verificamos que os valores ficam acima de 70. Esses resultados demonstram que controlando-se a admissão de fluxos de forma a não saturar a rede, os valores do atraso, jitter e perda de pacotes são mantidos em valores toleráveis que viabilizam a utilização da arquitetura via satélite utilizando redes em malha como infraestrutura de acesso para VoIP.

O baixo número de fluxos VoIP suportados pelo $802.11 \mathrm{~b}$ pode ser contornado pela utilização de várias técnicas de otimização [12], ou pela utilização de padrões com taxas maiores, como o 802.11g. Nesse estudo consideramos a capacidade máxima da rede igual à capacidade efetiva da camada MAC. Entretanto, na prática isso não ocorre devido às interferências externas que podem inclusive variar a qualidade do link entre os nós. Todavia, isso não invalida o
CCA, no qual o tempo de ocupação do canal é obtido a partir de informações coletadas em cada nó da rede de acesso.

\section{CONCLUSÕES}

Nesse estudo, consideramos a utilização de uma rede em malha IEEE 802.11, associada a um enlace de satélite, para prover infra-estrutura de acesso VoIP para pequenas comunidades remotas. Através de medidas efetuadas em um enlace de satélite e de uma métrica para avaliação da qualidade da voz, demonstramos que o enlace de satélite impõe requisitos estritos de $\mathrm{QoS}$ à rede de acesso.

Através de simulações efetuadas com o NS-2, e considerando os resultados das medidas efetuadas no enlace de satélite, demonstramos que através de um mecanismo efetivo de controle de admissão é possível a utilização de uma rede de acesso em malha, associada a um enlace de satélite para o transporte de VoIP para comunidades remotas de baixa densidade populacional.

Propomos um mecanismo de controle de admissão, centralizado no gateway de acesso ao enlace de satélite, capaz de restringir o acesso de novas chamadas, mantendo a qualidade das chamadas existentes, enquanto maximiza o número de chamadas admitidas. $\mathrm{O}$ mecanismo decide se aceita ou não uma nova chamada, baseado em medidas efetuadas em cada nó da rede de acesso em malha.

Como trabalhos futuros, pretendemos comparar os resultados das simulações com testes práticos utilizando um enlace de satélite. Também, pretendemos implementar um esquema de controle de violação de QoS e um mecanismo de diferenciação de tráfego centralizado, permitindo que a rede seja utilizada para fornecer também acesso a internet.

\section{REFERÊNCIAS}

[1] MELLO, T. e KIEFER, A. Satellite VoIP Access Gateway. International Conference on Digital Telecommunications (ICDT'06), 2006.

[2] SISALEN, D. et al,VDSat: Nomadic Satellite-Based VoIP Infrastructure. 2nd International Symposium on Wireless Communication Systems, p.619-623, 2005.

[3] PGMU, Plano Geral de Metas para Universalização. Decreto ANATEL 4.733, junho 2003. http://www.planalto.gov.br/ccivil_03/decreto/ 2003/D4733.htm. Acesso em: 28 de janeiro de 2008.

[4] DVB Forum, http://www.dvb.org. Acesso 20 de março de 2008.

[5] ITU-T Recommendation G.107, The E-Model, a computational model for use in transmission planning. ITU-T, March 2005.

[6] ITU-T Recommendation G.108, Application of the E-model: A planning guide. ITU-T, September 1999.

[7] J. Jun and ML Sichitiu, "The Nominal Capacity of Wireless Mesh Networks," IEEE Wireless Communications 2003.

[8] J. Jun, P. Peddabachagari, and M. L. Sichitiu, "Theoretical maximum throughput of IEEE 802.11 and its applications," IEEE International Symposium on Network Computing and Applications, Apr. 2003.

[9] Website oficial do NS-2, http://www.isi.edu/nsnam/ns/. Acesso $20 \mathrm{de}$ março de 2008 .

[10] Y. Yang, R. Kravets, Contention-aware admission control for ad hoc 1574 networks, IEEE Transactions on Mobile Computing 2005.

[11] KIM, Kyungtae; KASHYAP, Anand e GANGULY, Samrat On Admission of VoIP Calls Over Wireless Mesh Networks. IEEE International Conference on Communications (ICC 2006).

[12] P. Hsiao, A Hwang, H Kung and D Vlah, "Load Balancing Routing for Wireless Access Network," IEEE INFOCOM 99.

[13] I.D. Chakeres, E.M. Belding-Royer, PAC: perceptive admission control for mobile wireless networks, in: Proceedings of QShine (Dallas, TX, October 2004). 